\title{
Innovative Gsm Bluetooth based Remote Controlled Embedded System for Irrigation
}

\author{
Indu Gautam \\ Department Of Electronics And Comunication \\ IGIT,IP University,Delhi,India
}

\author{
S.R.N Reddy, phD. \\ Department Of Electronics And Comunication and \\ Computer Science \\ IGIT,IP University,Delhi,India
}

\begin{abstract}
In past few years controlling and monitoring the machines remotely has seen an interesting field of study among researchers. This paper mainly focuses on reviews in the field of remote monitoring and control, the technology used and their potential advantages. The paper proposes an innovative GSM/Bluetooth based remote controlled embedded system for irrigation. The system sets the irrigation time depending on the temperature and humidity reading from sensors and type of crop and can automatically irrigate the field when unattended. Information is exchanged between far end and designed system via SMS on GSM network. A Bluetooth module is also interfaced with the main microcontroller chip which eliminates the SMS charges when the user is within the limited range of few meters to the designated system. The system informs users about many conditions like status of electricity, dry running motor, increased temperature, water content in soil and smoke via SMS on GSM network or by Bluetooth.
\end{abstract}

\section{Keywords}

Bluetooth, GSM, irrigation, SMS.

\section{INTRODUCTION}

Global system for mobile communication (GSM) is one of the most trustable wireless communication system that can be accessed and used very easily. The price of it transreceiver module and subscription fee of it's services is very low so it is very cost effective also. Embedded system interfaced with GSM module can widen the scope of embedded design and enhanced the application areas of controlling and monitoring systems to a great extent. During the past decade network services has extended beyond speech communication to many other custom specified embedded design application. This paper proposes an innovative GSM-Bluetooth based remote controlled embedded system for irrigation. The interface and communication between user and designed system is via SMS on GSM network or by Bluetooth if the user is within the range of $10 \mathrm{~m}$ of designed system.

India is a country of agriculture and it is backbone of Indian economy. Irrigation is heart of agriculture. Irrigation is used to assist growing crops in the field land during the inadequate rainfall period. Pesticide is used preventing, destroying or mitigating any pest. Both of these are very important for good productivity and both need time to time application in the farm field. In India approximately $20 \%$ of farmers are dependent on electric water pumps for irrigation in their field.

There are many problems associated with irrigation
- Normally agricultural lands are very far from the farmer's house so farmers have to go farm land for irrigation that causes inconvenience and fuel consumption(if used any vehicle).

- Farmers are enable to know the status of electricity at the farm field as the nature of supply of electricity is quite unpredictable.

- There are frequent instances of burning of motor due to unpredictable voltage fluctuations and dry running.

- There is possibility of presence of venomous element in farm field.

- Farmers are present in farm field when pesticides are sprayed. These pesticides are very harmful for farmer's health.

All these issues are handled in the proposed system. The system will send status of power supply via Bluetooth/SMS on GSM network to user. The system will check the water flow from the pump. If electricity is there but no water supply is available, system will send information to user via Bluetooth/ SMS on GSM network. Temperature sensors and humidity sensors installed in the field take the measurement of humidity level of soil and environmental temperature and send this information to users. The user sends data in the form of SMS on GSM network to start or stop the irrigation according to received information.

The smoke sensor sends emergency information to user in case of fire in field or burning of motor. The use of Bluetooth module in the proposed design made the design low power, low cost, small size and robust. Bluetooth is a highly versatile and attractive technology among other short range wireless technology available in market. Bluetooth is an unlicensed technology that operates globally at $2.4 \mathrm{Ghz}$. It can link different digital devices within a range of $10 \mathrm{~m}$ to $100 \mathrm{~m}$ at a speed up to $3 \mathrm{mbps}$. This paper gives a review of various work done in the field of remote monitoring and control, the technology used and there potential advantage for agriculture irrigation as a specific application area. With this also proposes an innovative GSM-Bluetooth based remote controlled embedded system for irrigation.

The proposed design has advantage of both GSM and Bluetooth technology and the system can be monitored and controlled by both, by using the SMS for remote monitoring and control and by using Bluetooth if the user is within a limited range and thereby reducing the usage charge of SMS on GSM network. The rest of the paper is organized as follows. Section II gives the work done in the field of remote monitoring and control system based on technology, processor used and advantages of the system. Section III analyzes the existing systems. Section IV describes the proposed system. Finally section V concludes the paper. 
The block diagram of the proposed system has been shown in figure 2. The system consists of 8-bit PIC microcontroller that is interfaced with different types of sensors. The PIC microcontroller has inbuilt ADC so no need of external ADC in the system. Depending on the reading from the temperature and humidity sensor and type of crop the designed system decides the on/off time of pump motor for precise irrigation. Information from all the sensors are send to end users via SMS on GSM network or via Bluetooth if the user is within $10 \mathrm{~m}$ range of designed system.

\section{CLASSIFICATION OF REMOTE CONTROL AND MONITORING SYSTEMS}

To understand the method and technique used for the design of a generic remote control and monitoring systems wide variety of remote control and monitoring systems based on different technologies for different applications have been studied [1][25]. From technical point of view this system can be classified on the basis of technology, processor used, tools, programming code etc. Table 1 shows the classification of existing system based upon different criteria.

\subsection{Technology used and Application}

Gao Guohong and Liu Yi introduced an application based on single chip computer (AT89S52) in agriculture and landscape irrigation system [1].The system has many functional units like clock unit ,alarm unit and the display module . This approach reduces the staff workload, improves effective resource utilization, increases crop productivity and thereby reducing the cost of agricultural products.

Vandana Dubey [2] proposed a wireless sensor network based remote irrigation control system and automation using DTMF code. In this approach, cell phone or landline phones are used for starting and controlling the irrigation and pesticide spraying. The implementation of wireless sensor network gives better result and thus makes the work very convenient and efficient.

A very efficient system that uses miscall/SMS for different message and command indication from system cell phone and user cell phones respectively was developed by Vasif Ahmed [3].This system uses Bluetooth for data communication between microcontroller system and system cell phone but with a range of $10 \mathrm{~m}$.In this work, the temperature sensor gives output directly in digital form and have error detection capability. The system has real time clock to provide timing information. The system results in uniform distribution of water in fields at regular interval of time, reduced Labour cost, minimization of occurrence of motor faults, efficient use of water resources. Intimation about completion of task to the user can be send through miscall/SMS. The system can be implemented to any cell phone model. The system is convenient and of low operating cost due to use of miscall compared to SMS.

Li Wenyan proposed a design of wireless water saving irrigation system based on solar energy [4].The system in work is composed of wireless sensor node, routing node and gateway node with lead acid battery that is recharged by solar cell panel. The main advantage of system is that sensor node can be added and deleted arbitrary and very easily and user can change the threshold of the irrigation according to the specific need of crop.
An advancement in the previous works is done where different sensor nodes and simple Functional nodes form a wireless network .This system was proposed by Zheng Yao and Guohaun Lou [5]. In this work, data collected by Zigbee wireless sensor network is transmitted to gateway node and PC through LAN or WAN. This system uses fuzzy neural network to control agricultural irrigation. The system provides optimal scheduling, improves productivity and better growing environment for crop.

Zeng Peng [6] introduced real time monitoring system for soil moisture content based on (ATmega128) microcontroller/OS-2 operating system serial data flash and time keeping chip, soil moisture sensor, RS-455 microcomputer and RS-232 transreceiver and a host computer. In this work, microcontroller unit with an operating system is introduced to enhance the performance irrigation and provide real time multitasking process capabilities. Advantage of system is that the user can set the work time on demand and around 15 year's data (with one sample one per hour) can be stored.

Lei Xiao [7] proposed a system that can real timely monitor the agriculture environment information using wireless sensor network. The system in work uses MSP430 microcontroller and CC2420 Wireless communication module. The main advantage of the system is that system is steady in performance and facilitated in operation.

Wireless sensor network for precise agriculture monitoring [8] developed for monitoring the western region special agriculture product has been proposed. The system in work has precision agriculture monitoring system that has WSN gateway and communication server. The system uses ATmega 128L microcontroller that supports Tiny OS software with AT86RF230 as radio frequency chip and AT45db041B as memory. The main advantage of system is that the user can access the website via graphical user interface and can easily get real time data send by the nodes and improve the productivity by managing and monitoring the growth period of the crop in farm land.

Another WSN based greenhouse monitoring and control system[9] where WSN, gateways, 14 sensor nodes, a management subsystem, 1 actuator node and 2 sink nodes are deployed in greenhouse. The system in work has WLAN APs that give long range wireless link between WSN and management sub system which is about $0.5 \mathrm{~km}$ far away from greenhouse. The system provides easy and user friendly interface to farmers with hand held devices like PDA and PC.

An advanced system based on WSN zigbee [10] and ARM processor has been proposed. The first layer of WSN node is made up of CC2430 chip integrated with 8051 microcontroller and 2.4 GHZ RF trans receiver. These nodes communicate with gateway nodes that are composed of CC2308 GPRS MODULE MC391 through RS232 bus. The system has advantage that it is low cost, flexible and has user friendly human-computer interface.

An advance system based on WSN that monitor environment condition for precise agriculture is proposed by Jianfa Xia[11]. The system y collects various environmental parameters using Telos B wireless sensor node that consist of MSP430F1611 and CC2420 chips. Data from these WSN is send to gateway node through RS232. System in work has a web based information transmitting and inquiring subsystem. The system uses Google map technique to give location information, green house 
environmental status and give real time voice and SMS alarm services. The whole system is powered by solar energy and storage batteries. The system is low cost and highly scalable. The system gives real time stable and accurate services for agriculture.

Most of the field control and monitoring system use PC and cell phones but Jzau Sheng Lin [12] proposed a system that use SOC platform as web server. The system uses zigbee technology based wireless sensor. The zigbee receiver has different sensors and a microcontroller unit (SPC061A) that polls each sensor quickly and receives the data. As the system use zigbee technique so it solved wireless transmission problem and thus replaces long cables of field signal monitoring system. The system used SOC (built in XILINX SPARTAN-3) that reduces cost and physical size of the whole system.

A WNS (wireless sensor node) based real time remote monitoring and warning system [13] has been proposed. The system is made up of wireless environment monitoring equipment (WEME) and mobile phone. WEME uses solar power for it's working and has multi pin data interface for connecting different sensors. The system has advantage of online information acquisition. The user can remotely enquire and can do real time monitoring.

Another system for remote sensing and control of an irrigation system using a distributed wireless sensor network [14] has been proposed. The system has in-field sensing stations for sensing different parameters of field land and weather station for sensing micro metro logical information. The system uses Bluetooth, TCP/IP technology for sending data wirelessly. The system uses user friendly decision making program (WISC) for controlled irrigation. The system gives a low cost solution for WSN.

Another zigbee based prototype used for irrigation control using wireless sensor and actuator network has been proposed [15]. The data is sent wirelessly through zigbee to the sink node. The system can transform the data in less time. The system provides addressing for sensor network and gives decision making and fault tolerance capability. The system is easy to deploy, cost effective and decreases the labor cost for watering.

An automatic irrigation system for rice cultivation is proposed by LL. Pfitcher [16]. The system has a supervisory system that use SCADA software for monitoring and control of irrigation pump and watering level. The transmitter module (DX80N2X2S2S) of sensor works at $2.4 \mathrm{GHZ}$. The wireless gateway (DX80G2M2S) receives the signal from sensors and sends the data to controller using MOD BUS Protocol. The system uses telephone communication through GPRS message to establish communication between controller (BCM2085B) and supervisory system. The system uses ultrasonic sensors (T30UFDNCQ)in field land.

As the system uses GPRS communication with DNP3 protocol this makes the system more reliable. System sends all warning conditions using alarm management feature of SCADA.

An another GSM based irrigation system [18] that uses solar energy to run motor which is controlled by PIC microcontroller (16F877A) has been proposed. The system can start/stop the pump and can provide water level information by sending SMS on GSM network. The main advantage of proposed system is that the cost of operation and maintenance of solar pump is negligible. The system can be operated from any place as it can be completely automized and works on highly reliable and efficient GSM network.

Rajeev G Vishwakarama [19] proposed a system where the farmer can monitor power on/off, voltage supply level of electricity and control up to 8 different devices by sending SMS on GSM network. The system uses GSM mobile phone that support AT command, AT-89C51 microcontroller, max 232 IC and LCD for monitoring the current reading of all the measured parameters. As the system does only on/off operation therefore for such applications AT-89C51 microcontroller is the best suited option among available one.

An another system of embedded wireless video monitoring system based on GPRS [20] has been proposed. Here image collection and compression is done on embedded Linux platform that support USB interface. The system uses Samsung's S3C2410 16/32 bit RISC microprocessor. To reduce total cost of the system the microcontroller includes separate 16 $\mathrm{KB}$ instruction, $16 \mathrm{~KB}$ data cache, MMU, LCD controller, Boot Loader, system manager, 3 channel UART, DMA, I/O port, RTC, ADC and touch screen interface. The system is highly reliable and widely used in the applications where there is requirement of high real time monitoring and control.

An another system based on GPRS technology with better application prospect [21] is proposed by Lu limei, Xu Lizhong. The system has sensor node to gathers hydrographic information and the sink node to receive the real-time data through the GPRS network. The system replaces the wired transmission with the wireless transmission, which reduces the installation and maintenance costs and improves the system reliability and efficiency to a great extend.

A Low cost soil moisture monitoring system is presented in [22]. The paper describes a PC controlled irrigation monitoring and controlling system with wireless communication. The design of the overall system provides consistent soil moisture measurements and allows automatic watering of the soil at a very low cost. PIC processor is used with Linux wireless system which used Amplitude Modulation to transmit data.

Another approach using GSM technology to communicate with the remote devices via SMS has been proposed [23]. This paper illustrates a technique for remotely reading electricity meter (both postpaid and prepaid) readings using SMS. As the data collection is based on SMS so the system is very quickly and efficiently and reliable.

A wireless sensor network (WSN) based system used for an intelligent temperature measurement system [24] has been proposed. The system in use has digital multipoint thermometers for temperature measurement. System uses an advanced RISC microprocessor (ARM) and Wireless Fidelity (Wi-Fi) technology for data transmission. A special data storage file system is accomplished for reading and writing SD card, as well as for the management of the data file by the FAT16 file system. Wireless sensor networks are easy to establish without using cables and offers a greater flexibility.

An another system on automation of free-standing greenhouse using supervisory control and data acquisition (SCADA) has been proposed. This system gives a kiosh type of approach to farmers. Kiosh monitors and governs entire green house operation. 
From the above discussion, it is concluded that designing a remote monitoring and control system that fulfills all the requirements simultaneously is a complicated task. Every proposed system has its own merits and demerits. However, still there is possibility of designing an innovative cost effective and high performance system that can work optimally in different applications. An innovative GSM-Bluetooth based remote controlled embedded system for irrigation is proposed in this paper.

\subsection{Processor used}

There is a long list of processors used in different applications of control and monitoring system. All the system [1][24] used different processors with different technology and in few system different processor are used for same technology.

[1][2] has implemented their design using AT89S52 processor . These processor are of 8 bit with 256 byte RAM, $8 \mathrm{~K}$ ROM and no EEPROM, ISP PWM, I2C and ADC.

[5][10][19][20][23] have implemented their designs using 8-bit microcontrollers of 8051 family. These processors do not have on chip $\mathrm{ADC}$ so $\mathrm{ADC}$ is connected externally in these designs.

[3] [6] [8] [24] [25] have implemented their designs using 8-bit Atmega Series AVR processors with different size of programmable flash memory. It also has in built ADC with 516 $\mathrm{k}$ EEPROM. It supports ISP mode and I2C BUS.

[4][7][11] used a 16-bit MSP (mixed signal processing) microcontroller. Its peripheral circuits include Liquid crystal display, keyboard functions, A/D converter and so on and it is suitable for applications that requires extremely low power consumptions.

[9] uses JN5121 which enables user to implement IEEE802.15.4 or Zigbee compliant systems with minimum time to market and at a minimum possible cost.

[12] has implemented their design using SPCO61A microcontroller that has $2 \mathrm{~K}$ words of SRAM , 32K owrds of Flash ROM Data memory,32 programmable IO ports ,7 channel of 10-bit Digital to Analog convertor and an In-Circuit -Emulator.

[13] has implemented their design using STC11L60XE microcontroller.which is a single-chip microcontroller and is based on a high performance architecture of 80C51.

[16] has implemented their design using BCM2085B microcontroller which has large no. of modules for serial communication ports.

[18][22] has implemented their design using PIC 16F877A processor which is a 40 pin IC. It a an 8-bit processor with 368 byte RAM, 8K ROM, 256 byte EEPROM, inbuilt ADC and support I2c.It does not support PWM. PIC controllers also integrate a number of system level functions and in this way significantly reduce the component count, board area and system cost to a great extend.

\section{ANALYSIS OF EXISTING SYSTEMS}

All these systems [1]-[24] are well suited for remote control and monitoring applications depending upon the requirement of the system.

PC based technology is explained in[3] [4] [5] [6] [8] [9] [10] [11] [12] [14] [16] [17] [20] [21] [22] [23] [24][25] .In this system, PC is the remote monitoring station and microcontroller is the main controlling device. Although one can monitor and control devices remotely from any part of the world provided internet access is available to user. The system is of high cost due to the requirement of a computer. And special hardware and software installation is required to control the devices in a particular design. It is difficult to monitor and control the status of different sensors and devices in case of power failure unless you have a battery backup which is an additional cost.

Bluetooth based solutions are also used for this purpose [3] [14] [25]Although Bluetooth eliminates the usage cost of the network to a great extent, main limitation of Bluetooth technology is its range of operation which is limited to a few meters. So one can remotely monitor and control devices using this technology but it is prerequisite for each controlling device design to have a dedicated Bluetooth module. As the single Bluetooth module is shared by several devices so it results in access delay. Interference is another very big problem of Bluetooth technology.

WNS based remote monitoring and control systems are explained in[7][8][9][10][11][22]. The wireless sensor network eliminates the need of hard wire sensor station across the farm land and in this way reduces the installation and maintenance cost. WSN

uses ad hoc network (mobile wireless network) that has advantages for agricultural applications because of its unique features like mobility and self configurability. But the communication distance is limited among wireless sensor nodes and ability of signal through wall is very poor.

A hard-wired system from in-field sensing station to monitoring station takes extensive time and costs to install and maintain. It is not practically feasible to hard wire the system for long distances and may also be not acceptable to farmers because it can

Interfere with normal farming operations. All these problems are solved by GSM based remote monitoring and control systems [2] [3] [13] [18] [19] [2] [23].

The systems where both PC and Mobile act as monitoring and control unit are given in [3] [8] [10][11][12][20][21][22] and [24]. PC acts as remote monitoring station and mobile unit controls everything remotely. These systems eliminate one of the drawbacks of real time monitoring and control using internet but it again increases fiscal cost due to PC which is again a drawback.

Another Zigbee based technology used for remote monitoring and control systems is given in [4][5][10][12][15]. It provides easy wireless installation of sensors at a lower cost, reasonable range and average data rate. Zigbee supports data rate of $250 \mathrm{kbps}$ which is more than enough for SMS, it is not intended for voice and data streaming because it consumes a large bandwidth and consumes power quickly and thus makes the technology unsuitable for real time applications.

From the above discussion it is concluded that designing a remote monitoring and control system that satisfies all the parameters simultaneously is a complicated task. Each proposed methodology has its own merits and demerits. However, there is still a possibility of designing a cost effective and efficient system which has an improved performance in most of the respects is a complicated task. Each proposed methodology has its own merits and demerits. However, there is still a possibility of designing a cost effective and efficient system which has an improved performance in most of the respects.

A new innovative GSM/Bluetooth based remote controlled embedded system for irrigation is proposed in the next section. 


\section{PROPOSED SYSTEMS}

The block diagram of the proposed system has been shown in figure 2. The system consists of 8-bit PIC microcontroller $(16 \mathrm{~F} 877 \mathrm{~A})$ that is interfaced with different types of sensors. The PIC microcontroller has inbuilt $\mathrm{ADC}$ so no need of external $\mathrm{ADC}$ in the system. The designed system has provision of keys for manually selecting the irrigation mode (for farm field / garden) and for spraying of pesticide in farm field/garden. The same operation can be done by sending SMS on the GSM network by the user. Depending on the reading from the temperature and humidity sensor and type of crop the designed system decides the on/off time of pump motor for precise irrigation. The system will send status of power supply via Bluetooth/SMS on GSM network to user. The system will check the water flow from the pump. If electricity is there but no water supply is available, the system will send information to user via Bluetooth/ SMS on GSM network. The system will raise an alarm and send message to user if there are any traces of smoke in the field land due to burning of motor or due to fire in the field land.

\subsection{System architecture}

The hardware of the system mainly includes a 8-bit microcontroller chip (PIC 16F877A), a GSM module, a Bluetooth module, RS232 interfaces and sensors. The microcontroller is interfaced with different sensors for controlling and monitoring different aspects of irrigation. Temperature sensor and humidity sensor are used to get the exact value of environmental temperature and level of water content in soil .Depending on the reading from the temperature and humidity sensor and type of crop the designed system decides the on/off time of pump motor for precise irrigation. Water flow sensor is used to check the condition of dry running of motor. The smoke sensors are used to check emergency conditions like fire in field or burning of motor. The analog data from temperature sensor is converted to digital using inbuilt $\mathrm{A} / \mathrm{D}$ converter. It provides this data to the microcontroller for analysis when requested and an alarm is raised in emergency conditions depending upon this data and an SMS on GSM network is send to the user's mobile. The measured values of sensors are displayed on the LCD. The GSM and Bluetooth modules which are the most important part of this designed system are interfaced with the microcontroller using a RS232 interface. The GSM module must have a SIM (Subscriber Identity Module) card to make the network identify to the user. The microcontroller communicates with the GSM module using the AT commands. These AT commands are used to send and receive SMS. The programming code for the microcontroller is written in some high level language $(\mathrm{C}$ language).

\section{CONCLUSION}

This paper proposes the design of the innovative GSMBluetooth based remote controlled embedded system for irrigation. The proposed system is a low cost system where information is exchange via SMS on GSM network. As we know the appropriate level of water in the farm field contributes to the quality of grains and highly affects the incidence of pests and diseases on crops. By using this system the farmers can get the information of electricity status at farm field, warning message like smoke in farm field due to fire or due to burning of motor in the farm field and the environmental temperature exceeds information from home or any where using a mobile phone. With this the farmer can switch on the motor for irrigation/pesticide spray from anywhere far from the actual field. The system has advantage of using Bluetooth if the user is within the $10 \mathrm{~m}$ range of designed system. The use of Bluetooth technology cuts down the cost of SMS if the user is within the limited range of designed system. The availability of the GSM network is a prerequisite for the system implementation. The system is highly beneficial for precise irrigation in farm fields and thus responsible for efficient utilization of water resource and men power.

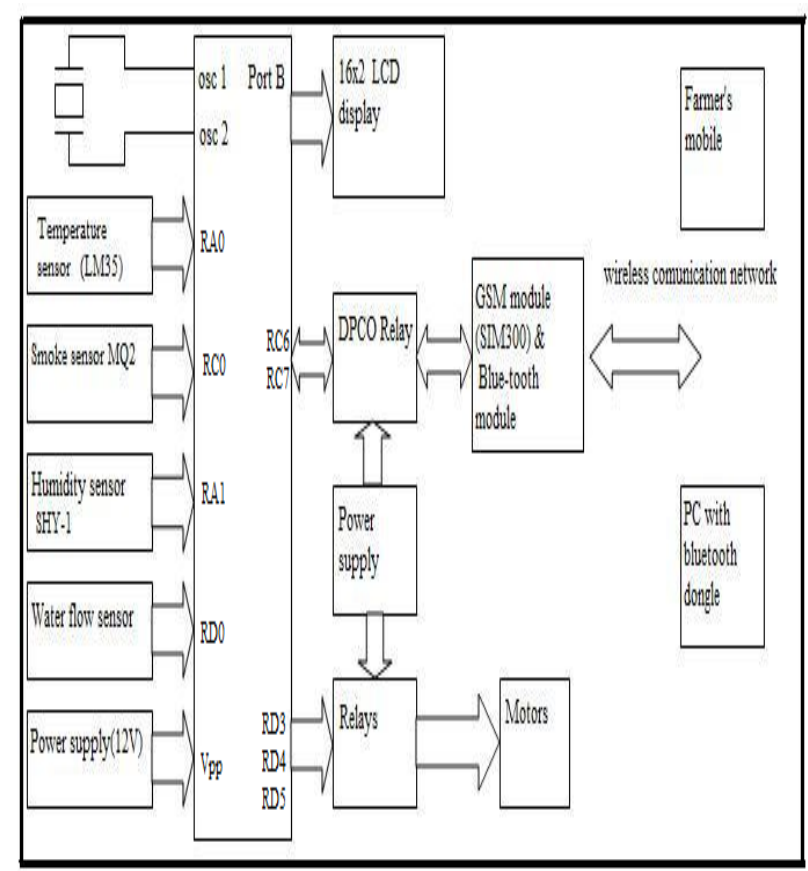

Figure 1: Block diagram of proposed system 
Table 1 Classification of Existing Remote Monitoring and Control Systems

\begin{tabular}{|c|c|c|c|c|c|}
\hline References & Technology & Processor & Monitoring station & Tools & Programming code \\
\hline [1] & Wired & AT89S52 & 7 segment display & Kiel IDE & Assembly \\
\hline [2] & GSM & AT89S52 & Mobile & Kiel IDE & Embedded in $\mathrm{C}$ \\
\hline [3] & GSM, Bluetooth & ATmega32 & Mobile, pc & $\begin{array}{l}\text { AVR studio, } \\
\text { Eclipse 3.2.2 }\end{array}$ & C, Java \\
\hline [4] & GPRS, Zigbee & MSP430F2274 & $\mathrm{PC}$ & C430 IDE & $\mathrm{C}$ \\
\hline [5] & LAN/WAN, Zigbee & 8051 & $\mathrm{PC}$ & Kiel IDE & Assembly \\
\hline [6] & Wired & $\begin{array}{l}\text { ATmega128 with } \\
\text { uc/os-II }\end{array}$ & PC or PDA & $\begin{array}{l}\text { uc/os-II ,AVR } \\
\text { Studio }\end{array}$ & $\begin{array}{l}\text { Visual } \mathrm{C}++, \mathrm{C}, \\
\text { Java }\end{array}$ \\
\hline [7] & WSN & MSP430 & PDA & C430 IDE & $\mathrm{C}$ \\
\hline [8] & $\begin{array}{l}\text { WSN,GPRS, } \\
\text { Internet }\end{array}$ & AT-mega128 & PC, mobile & AVR Studio & $\mathrm{C}$ \\
\hline [9] & $\begin{array}{l}\text { WSN,LAN, } \\
\text { Internet }\end{array}$ & JN5121 & PDA, PC & Kiel IDE & Java, interactive C \\
\hline [10] & $\begin{array}{l}\text { WSN,Zigbee } \\
\text { GPRS }\end{array}$ & 8051, S3C2410 & Mobile, PC & $\begin{array}{l}\text { Win CE5.0, Kiel } \\
\text { IDE }\end{array}$ & $\begin{array}{l}\text { Embedded visual } \\
\mathrm{c}++4.0\end{array}$ \\
\hline [11] & $\begin{array}{l}\text { WSN,GPRS } \\
\text { Internet }\end{array}$ & MSP430F1611 & Mobile, PC & $\begin{array}{l}\text { Tiny OS } 2.1 .0 \\
\text { Google } \\
\text { visualization API }\end{array}$ & - \\
\hline [12] & Zigbee & SPCO61A & PC, Smart phone & $\begin{array}{l}\text { XILINX } \\
\text { SPARTAN } 3\end{array}$ & $\mathrm{C}$ \\
\hline [13] & GSM & STC11L60XE & $\begin{array}{l}\text { OCM12864, } \\
\text { mobile }\end{array}$ & - & - \\
\hline [14] & Bluetooth & PLC & $\mathrm{PC}$ & $\begin{array}{l}\text { Wireless in field } \\
\text { sensing and control } \\
\text { software }\end{array}$ & $\begin{array}{l}\text { Microsoft visual } \\
\mathrm{c}++, \text {.net }\end{array}$ \\
\hline [15] & Zigbee & - & - & $\begin{array}{l}\text { Wireless sensing } \\
\text { actuator node } \\
\text { software }\end{array}$ & - \\
\hline [16] & GPRS & BCM2085B & $\mathrm{PC}$ & SCADA & - \\
\hline [17] & Wired & Z-80 & $\begin{array}{l}7 \text { Segment display, } \\
\text { PC }\end{array}$ & Delphi & $\begin{array}{l}\text { Assembly language } \\
\text { embedded in c }\end{array}$ \\
\hline [18] & GSM & $16 \mathrm{~F} 877 \mathrm{~A}$ & Mobile & MPLAB IDE & $\mathrm{C}, \mathrm{UML}$ \\
\hline [19] & GSM & AT-89C51 & Mobile & Kiel IDE & Assembly \\
\hline [20] & GPRS, Ethernet & S3C2410 & Mobile, PC & LINUX OS & - \\
\hline [21] & GPRS & C8051F310 & Mobile, PC & Kiel IDE & Pythan \\
\hline [22] & GSM,WSN & $\begin{array}{l}\text { PIC16F74(DIP } \\
\text { Configuration) }\end{array}$ & Mobile, PC & $\begin{array}{l}\text { AVR Studio, } \\
\text { MPLAB IDE }\end{array}$ & $\mathrm{C}$ \\
\hline [23] & GSM & 8051 & $\mathrm{PC}$ & $\begin{array}{l}\text { Kiel IDE, Linux } \\
\text { OS }\end{array}$ & Assembly, C ,Java \\
\hline
\end{tabular}




\begin{tabular}{|l|l|l|l|l|l|}
\hline$[24]$ & Wi-Fi & AVR & Mobile, PC & Kiel IDE & Assembly \\
\hline$[25]$ & Bluetooth & Atmega 64 & PC & AVR Studio & C \\
\hline
\end{tabular}

\section{REFERENCES}

[1] Gao Guohong, Liu Yi ,Fu Junhui and Wang Yingjun "Design of time control irrigation system based on single-chip computer"-2010 second international conference on communication systems, networks and applications.

[2]Wireless sensor network based irrigation control system and automation using DTMF code-2011 international conference on communication systems and network technologies.

[3] Innovative cost effective approach for cell phone based remote controlled embedded system for EEPROM is used for recording the data provided irrigation- 2011 international conference on communication systems and network technologies.

[4] Design of wireless water - saving irrigation system based on solar energy- 978-1-4577-0860-2/11/\$26.00 c 2010 IEEE.

[5] Research and development precision irrigation control system in agriculture- 2011 international conference on computer and communication technologies in agriculture engineering.

[6] Real-time monitoring system for soil moisture content based on uc/os-II operating system-978-1-4577-08602/11/\$26.00 c 2010 IEEE.

[7] Lei Xiao and Lejiang Guo"The realization of precision agriculture monitoring system based on wireless sensor network"-2011 international conference on computer and communication technologies in agriculture engineering.

[8]Wireless sensor network for precise agriculture monitoring-2011 fourth international conference on intelligent computation technology and automation.

[9]Study on precision agriculture monitoring framework based on WSN.

[10 ]Design of wireless monitoring and warning system for protected agriculture for environment- 978-1-4244-37092/10/\$25.00 c 2010 IEEE.

[11] Jianfa Xia,"An environment monitoring system for precise agriculture based on wireless sensor networks"2011 seventh international conference on mobile Ad-hoc and sensor network.

[12] Jzau-Sheng Lin and Chun-Zu Liu "A monitoring system based on wireless sensor network and an SOC platform in precision agriculture"- $200811^{\text {th }}$ IEEE international conference on communication technology proceedings.

[13] Zhuanwei Wang ,Chunjiang Zhao and Haihui Zhang” real-time monitoring and warning system in general agriculture environment"-2011 international conference of information technology, computer engineering and management science.

[14] Yunseop Kim,Robert G. Evans and William M. Iversen"Remote sensing and control of irrigation system using a distributed wireless sensor network"- IEEE transactions on instrumentation and measurement vol.57.no.7.july 2008.

[15] Ghulam Ali, Abdol Wahid Shaikh, Aqeel-ur-Rehman and Zubair A Shaikh "A framework for development of cost effective irrigation control system based on wireless sensor and actuator network for efficient water management"- 2010 second international conference ob mechanical and electronics engineering.

[16] L.L.Pfitscher,"An automated irrigation system for rise cropping with remote supervision"- preceding of 2011 international conference on power engineering, energy and electrical devices.

[17]Mr.Azzouz Benzekri and Refoufi'Design and implementation of a microprocessor based interrupt driven control for an irrigation system".

[18] Mr. K.Ganesh and Mr. S. Girisha"Embedded controller in farmers pump by solar energy"- 2011 international conference on recent advancement in electrical, electronics and control engineering.

[19] Rajeev G Vishwakarma and Vijay Choudhary,"Wireless solution for irrigation in agriculture" - proceedings of 2011 internationalconference on signal processing, communication, computing and network technology.

[20] Zhao Ji-Chun and Sun Su-Fen Yu Feng Luan Ru-Peng Zhang Wei," The design and realization of embedded wireless video monitoring system based GPRS".

[21] Yan Xijun, Lu limei, Xu Lizhong,"The Application of wireless sensor network in the Irrigation Area Automatic System", International Conference on Networks Security, Wireless Communications and Trusted Computing 2009, pp.21-24.

[22] Jeng-Nan Juang, R. Radharamanan; "Low Cost SoilMoisture System: A Capstone Design Project", International Conference on Intelligent Computation Technology and Automation, 2010, pp. 1012-1014.

[23] Md. Wasi-ur-Rahman, Mohammad Tanvir Rahman, Tareq Hasan Khan and S.M. Lutful Kabir,"Design of an Intelligent SMS based Remote Metering System", Proceedings of the IEEE International Conference on Information and Automation, 2009, pp. 1040-1043.

[24] Sankar, P., Norman, S.R., "Embedded System for Monitoring Atmospheris Weather Conditions Using Weather Balloon", International Conference on Control, Automation, Communication and Energy Conservation 2009, pp 1-4 June 2009.

[25] Bhutada, S.; Shetty, S.; Malye, R.; Sharma V.;Menon, S.;Ramamoorthy, R, "Implementation of a fully automatedgreenhouse using SCADA tool like LabVIEW", AdvancedIntelligent Mechatronics. Proceedings, 2005 IEEE/ASMEInternational Conference on, pp. 741-746, 2005 